\title{
Relative preservation of lumbrical versus thenar motor fibres in neurogenic disorders
}

\author{
SK YATES, R YAWORSKI, WF BROWN \\ From the Department of Clinical Neurological Sciences, University Hospital, London, Ontario, Canada
}

SUMMARY At late stages of median nerve entrapment in the carpal tunnel there may be total denervation of the thenar muscles. Surface electrodes over the thenar endplate zone may record an initial and predominantly positive " $M$ " response to supramaximal median nerve stimulation. By a combination of techniques, it has been established that this surface positive response originates from surviving lumbrical muscles innervated by the median nerve. Their relative preservation may be related to their location in the median nerve. Intraoperative investigations have shown that the thenar motor fibres are primarily located in the ventral-lateral part of the median nerve whereas lumbrical motor fibres are in a more dorsal location and hence are probably better protected against the flexor retinaculum. This relative preservation of lumbrical motor fibres has been observed in other peripheral neuropathies and motor neuron disease, but not in median nerve regeneration following nerve transection. The importance of the observation lies in the more precise localisation of the voltage source for " $\mathrm{M}$ " response in terminal or near-terminal carpal tunnel median nerve entrapments and avoidance thereby of possible misinterpretation of electrophysiological observations in this most common nerve entrapment syndrome.

The median nerve most commonly innervates the abductor pollicis brevis, opponens, part of the flexor pollicis brevis and the first two lumbrical muscles ( $\mathrm{L}_{1}$ and $\mathrm{L}_{2}$ ) in the hand. ${ }^{1}$ It has been claimed that the respective motor fascicles to the thenar and lumbrical muscle groups are located close together in the ventral half of the median nerve. ${ }^{1}$ Others, however, observed that partial transections of the median nerve could totally denervate the thenar muscles, leaving the lumbrical muscles intact. $^{2}$ This observation was interpreted to mean that the lumbrical fascicle occupied a more dorsal position in the median nerve. The innervation of the lumbrical muscles and relative positions of the lumbrical and thenar motor fascicles in the median nerve are important anatomical points to recall in the examination of patients with median nerve entrapments beneath the flexor retinaculum or other neurogenic disorders involving the innervation of the hand muscles. In median nerve transections total denervation of the thenar muscle group is not evidence, by itself, of complete transection of all the motor fibres in the median nerve. The innervation of

Address for reprint requests: Dr WF Brown, Dept of Clinical Neurological Sciences, University Hospital, 339 Windermere Road, London, Ontario, Canada, N6A 5A5.

Accepted 10 March 1981 the lumbrical muscles must be checked. Moreover, when a transected median nerve has been surgically re-joined the lumbrical muscles may be re-innervated prior to the thenar muscles. ${ }^{3}$

The above and other examples of the importance of the median innervation to the lumbrical muscles led to this investigation of the thenar and lumbrical innervation patterns in a variety of neurogenic disorders in the upper limb.

\section{Methods and patients}

Patients in the survey suffered from carpal tunnel entrapment (19), median nerve transections (2), recurrent thenar branch transection (1), thoracic outlet compressions (3), C5-6 large disc protrusion (1), and motor neuron disease (5). All median nerve entrapments were characterised by wasting, little or no detectable action of the abductor pollicis brevis and sensory loss in the distributions of the median nerve in the hand. All thoracic outlet patients had clinical characteristics typical of the patients described by Gilliatt. ${ }^{4}$

\section{ELECTROPHYSIOLOGICAL STUDIES}

Surface and intramuscular (DISA 13L58) electrodes were employed. The latter were used to detect evidence of denervation by looking for the presence 
of fibrillation potentials, positive sharp waves and changes in motor unit potentials characteristic of re-innervation. The end-plate zones for the thenar and lumbrical muscles were located by positioning the surface stigmatic electrode (Copeland-Davies) at a location where the initial voltage deflection of the " $M$ " response was negative, the peak-to-peak voltage (p-pV) maximum, and the rise time of the initial negative deflection the briefest. Beneath this position, miniature end-plate potentials and bi-phasic negativepositive potentials could usually be detected by an intra-muscular electrode. If, with the surface electrode, the thenar " $M$ " response was initially surface positive, positive-negative or no response was detected despite full exploration of the surface of the thenar muscles with the stigmatic electrode, the stigmatic electrode was moved to the flexor surface of the palm. Invariably the maximum p-pV and an initial negative " $M$ " response was observed when the stigmatic electrode was positioned over the first or second lumbrical muscle. When the origin for the surface potentials was in doubt, the intramuscular electrode helped to resolve the issue by determining whether local electrical activity was detectable in the muscle beneath the surface electrode.

The sequence illustrated in the table, beginning at the top, was used to determine the electrogenic origin of the " $M$ " response to stimulation of the median nerve at the wrist and to exclude spread of the stimulation to the ulnar nerve. It was important to exclude extension of the electrical stimulus to the nearby ulnar nerve to avoid any contribution to the surface recorded " $M$ " response from nearby or remote ulnar nerve innervated muscles. This was a particular risk when the threshold for excitation of the abnormal median nerve was much higher than the nearby healthy ulnar nerve (see also fig 4). A possible ulnar source was excluded by recordings at the same time from ulnar-innervated hand muscles with an intramuscular electrode and from the fifth digit with bi-polar ring electrodes. Extension of the stimulus to the ulnar nerve was betrayed by an EMG response in ulnar innervated hand muscles and detection of an antidromic sensory nerve action

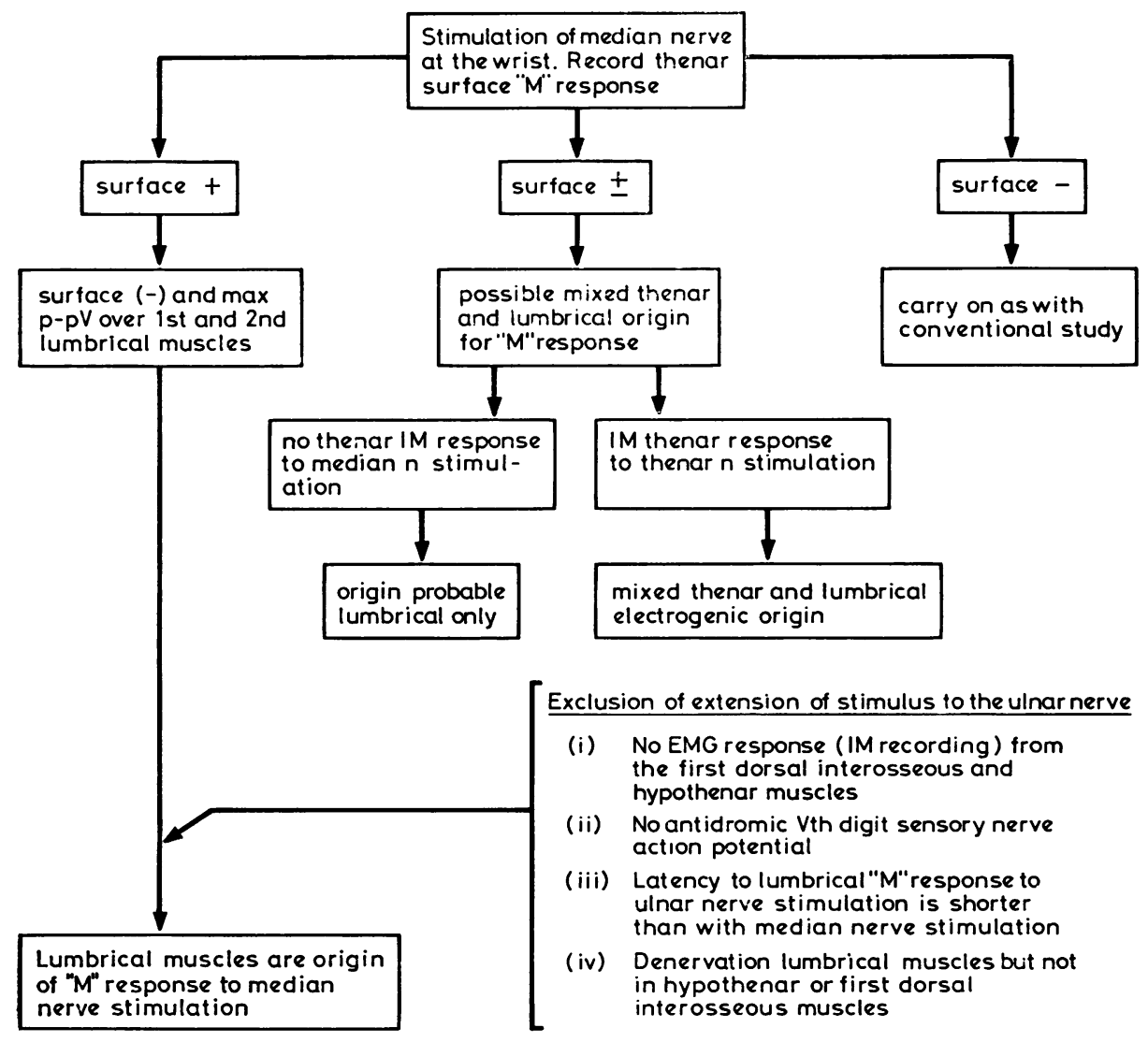


potential from the fifth digit.

Further complicating the matter is the fact that the lumbrical muscles are surrounded by muscles innervated by the ulnar nerve. The ulnar nerve could be responsible for an initial negative " $M$ " response over the surface of the lumbrical muscles if the stimulus to the median reached a level above threshold for the ulnar nerve. In the median nerve entrapments, however, the latency to the lumbrical " $M$ " response evoked by the stimulus to the median nerve was invariably longer than the latency to nearby ulnar innervated muscles, when the ulnar nerve was stimulated at the wrist level. Other tests for median nerve entrapment, thoracic outlet and other neurogenic disorders involved standard techniques and need not be repeated here. ${ }^{6}$

\section{Results}

CARPAL TUNNEL ENTRAPMENTS

Eighteen of the nineteen patients in this category had severe thenar wasting. In sixteen, median sensory nerve action potentials were not detected above the noise level of the system (1 microvolt, electronic averaging techniques were not used). In the other

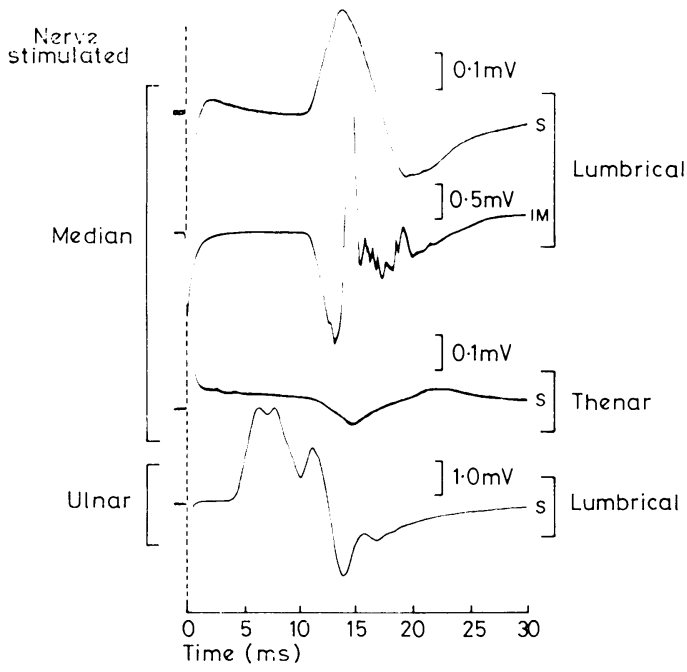

Fig 1 Illustration taken from a patient with median nerve entrapment beneath the flexor retinaculum. On the top are illustrated the surface(s) and intramuscular (IM) records from $L_{1}$ and $L_{2}$ in response to supramaximal median nerve stimulation. The same stimulus delivered to the median nerve evoked only an initial positive response in the thenar surface record and no intramuscular thenar EMG activity was detected. At the $L_{1}$ and $L_{2}$ surface electrode position, ulnar nerve stimulation evoked a much larger potential with a much shorter latency (bottom). three patients, the sensory potentials were abnormally low in voltage and the latencies prolonged. These nineteen patients represented less than five $\%$ of the total carpal tunnel population tested in this laboratory over a two and a half year period. Eleven of the nineteen patients had total denervation of the thenar muscles innervated by the median nerve. Either no thenar surface response or a surface positive potential was observed in response to median nerve stimulation and there were no local potentials. Furthermore, an intramuscular electrode located in the thenar muscles revealed no local motor unit potentials when the thumb was abducted or the median nerve was stimulated.

In the other eight patients, surface positivenegative responses were recorded over the thenar muscles (fig 2). The latency to onset of the initial positive deflection was invariably the same as the

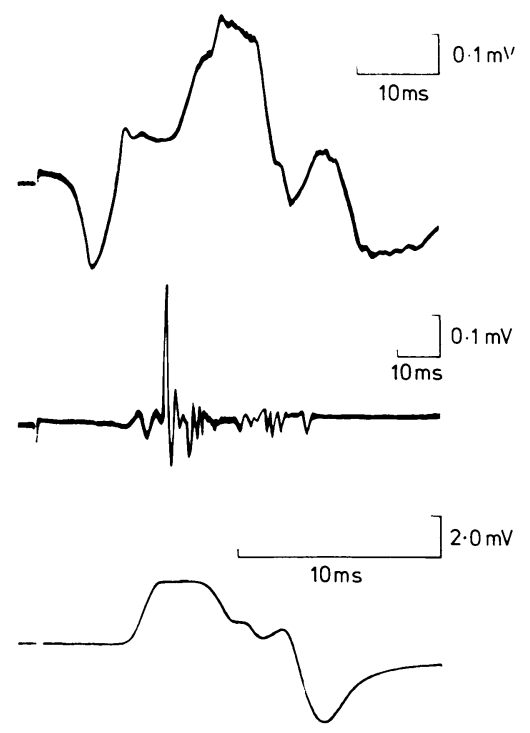

Fig 2 Illustration taken from a patient with a severe median nerve entrapment beneath the flexor retinaculum. Upper: Potential recorded by a surface electrode over the thenar muscles in response to supramaximal median nerve stimulation. Despite changes in the position of the stigmatic electrode, the initial response was positive. This was followed by later negative responses. Middle: The later thenar negative potential probably originated from the long latency thenar motor unit discharges here observed with an intramuscular thenar electrode when the median nerve was stimulated. Lower: Surface electrode record over $L_{1}$ and $L_{2}$, illustrating the initial negative deflection and the probable voltage source for the short latency initial positive potential recorded over the thenar muscles when the median nerve was stimulated (see upper). 
latency to onset of the lumbrical " $M$ " response; the later negative component of the thenar recorded surface response usually corresponding in timing to electrical activity generated by the thenar muscles themselves and detected by the intramuscular electrode (fig 2). In two of these patients a thenar " $M$ " response was detected only by the intramuscular electrode. In those patients with EMG responses in both the lumbrical and thenar muscles, the " $M$ " response voltage was larger and the terminal latency shorter in the lumbrical compared to the thenar muscles when the median nerve was stimulated at the wrist. The first and second lumbrical muscles were not normal, however, in any of the median nerve entrapments. Fibrillation potentials and positive sharp waves were detected in all patients in these muscles. In addition, in nine of the nineteen median nerve entrapments, there were fewer then ten lumbrical motor units detected by incremental increases in the stimulus intensity. This represents less than $10 \%$ of the normal number of motor units estimated by anatomical techniques in these muscles. ${ }^{7}$ Loss of lumbrical motor units was further suggested by the observation that the surface $\mathrm{p}$-pV of the lumbrical " $M$ " response was less than 1.0 $\mathrm{mV}$ in ten of the patients. In healthy controls, p-pV is at least $5 \mathrm{mV}$.

\section{TRANSECTION OF THE RECURRENT THENAR} MOTOR BRANCHES

In this patient the recurrent motor branch was transected by a puncture wound. The result was total denervation of the thenar muscles, but no denervation in the lumbrical muscles. Sensory nerve action potentials in the median nerve territory were normal. The maximum " $M$ " voltage was $6.4 \mathrm{mV}$ p-pV recorded on the surface over the lumbricals. The terminal latency was $4.5 \mathrm{~ms}$ (distance cathode to stigmatic electrode was $85 \mathrm{~mm}$ ). This patient provided us with the only opportunity so far of learning what the contribution of the first two lumbricalmuscles were to the " $M$ " response recorded on the surface when there was no contribution from the thenar muscles.

\section{THORACIC INLET COMPRESSIONS}

Three patients met the clinical criteria for the thoracic inlet compression described by Gilliatt. In all three, the thenar muscles innervated by the median nerve were denervated and only a surface positive response was recorded over the thenar muscles when the median nerve was stimulated at the wrist. The same stimulation, however, evoked a negative " $M$ " response over the first and second lumbrical muscles. In all three patients the " $M$ " response was less than $1.0 \mathrm{mV} \mathrm{p}$-pV and denervation

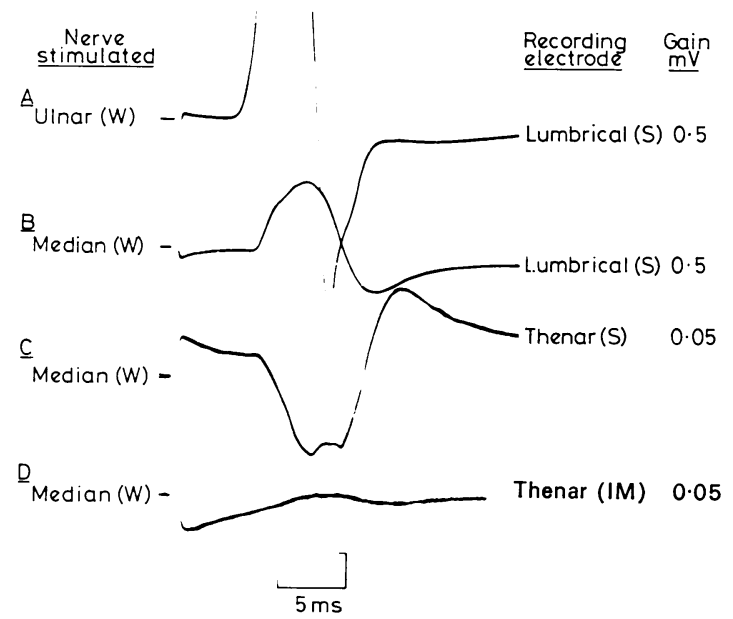

Fig 3 Illustration of the surface $(s)$ and intramuscular (IM) " $M$ " responses to ulnar or median nerve stimulation recorded in a patient with entrapment of the median nerve beneath the flexor retinaculum. There was total denervation of median innervated thenar muscles. Note the absence of any motor unit spike activity in the thenar muscles with supramaximal median nerve stimulation $(D)$ and the corresponding large surface positive-negative response recorded with the surface electrode over the thenar muscles at the same time $(C)$. The "sink" for this thenar surface positive response was located over $L_{1}$ and $L_{2}(B)$. Ulnar nerve stimulation at the level of the wrist $(W)$ evoked a shorter latency but larger voltage " $M$ " response at the same lumbrical recording electrode position $(A)$.

potentials were detected in the lumbrical muscles in addition to denervation in other hand and forearm muscles innervated by the ulnar nerve.

\section{MEDIAN NERVE TRANSECTIONS}

In two patients there was clear evidence of relative preservation of the lumbrical innervation in the presence of total denervation thenar muscles innervated by the median nerve. Denervation potentials were detected in the first and second lumbrical muscles. In one patient examined three months following surgical reunion of a previously completely transected median nerve, re-innervation of the thenar muscles was not detected but three motor units were detected in the lumbrical muscles. At the next examination, two months later, evidence of re-innervation of the thenar muscles was present but over the two subsequent examinations which extended over the next four months, the lumbrical " $M$ " response remained larger than a thenar " $M$ " response and had the shorter latency. This patient illustrates the fact that the lumbrical muscles may be 


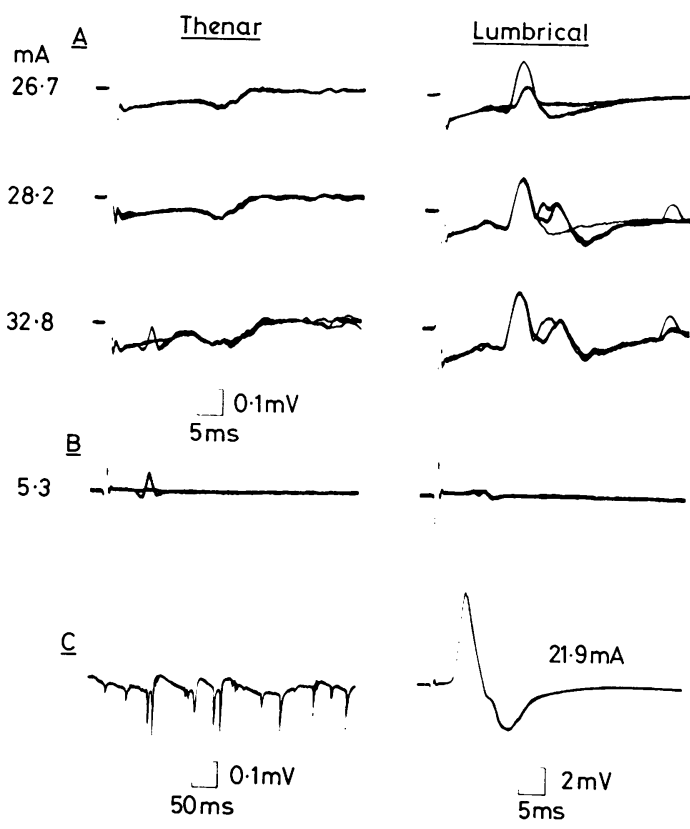

Fig 4 Illustration taken from a patient whose median nerve was transected just proximal to the wrist and resutured 4 months earlier. (A) Stimulation of the median nerve at increasing stimulus intensities, to illustrate that only a surface positive was recorded at 26-28 ms, the same intensities evoking 5 all or nothing motor unit potentials with initial negative deflections over $L_{i}$ and $L_{2}$ (surface electrodes both thenar and lumbrical). When the stimulus intensity was increased still more, a short latency all or nothing unit response was recorded just prior to the thenar surface positive response but no additional lumbrical motor units were recruited. The origin for the latter short latency all or nothing motor unit response was from the nearby flexor pollicis muscles. See (B) . (B) Threshold stimulation of the ulnar nerve at the level of the wrist and recording with the surface electrode over the thenar muscles. The first motor unit recruited from the flexor pollicis muscle was the same as the unit recruited by much higher stimulus currents delivered to the median nerve. This proved there had been extension of the stimulus current from the stimulating electrode placed over the median nerve to the adjacent ulnar nerve. (C) Positive sharp waves recorded from $L_{1}$ and $L_{2}$ (left). A much larger potential was recorded by the $L_{1}$ and $L_{2}$ surface electrode in response to supramaximal stimulation of the ulnar nerve at the wrist (bottom right).

the first muscles re-innervated. They are, therefore, important muscles to check when looking for the earliest evidence of motor fibre regeneration in median nerve transections.

\section{CERVICAL DISC PROTRUSION WITH THENAR WASTING}

In this patient the clinical examination pointed to a probable thoracic inlet compression of the type discussed by Gilliatt.4 ${ }^{5}$ However, no abnormal cervical rib was present. Instead, a large centrolateral disc protrusion was present at the C5-6 level. Sensory nerve action potentials in the median nerve territory were normal. The antidromic fifth digit sensory nerve action potential nevertheless was abnormally low in voltage, though the terminal latency was in the normal range. Frequent fibrillation potentials and positive sharp waves were recorded in the thenar, first dorsal interosseous, and adductor pollicis muscles. Fewer fibrillation potentials and positive sharp waves were observed in the hypothenar and flexor carpi ulnaris muscles. In response to supramaximal stimulation of the median nerve, only a small surface positive potential was detected over the first two lumbrical muscles. In contrast, a low voltage but surface negative response (two motor unit potentials) was recorded over the thenar muscles. Here, therefore, the lumbrical

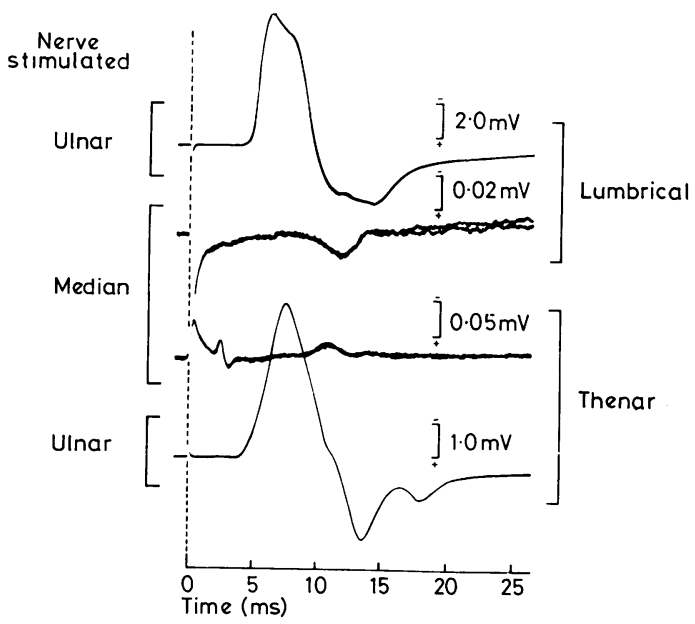

Fig 5 Illustration taken from a patient with a large C5-6 disc. In this patient only a small surface positive response was recorded by the $L_{1}$ and $L_{2}$ surface electrode when supramaximal stimuli were delivered to the median nerve (second trace from top). The same stimulation evoked a small negative response over the thenar muscles (third trace from the top). The last corresponded to the only two all-or-nothing motor unit potentials which could be excited by median nerve stimulation.

Note the normal latency and voltage of the sensory pre-potential. Ulnar nerve stimulation, by contrast, evoked much larger voltage potentials over both the lumbrical region (top trace) and the thenar muscles (bottom trace). 
muscles were totally denervated at a time when some residual innervation of the thenar muscles was yet present.

\section{MOTOR NEURON DISEASE}

In our experience it has not been uncommon to see patients with motor neuron disease at a later stage of atrophy of the upper limbs, who have total denervation of the thenar muscles but retain partial innervation of the lumbrical muscles. Preceding the surface positive response may be a sensory prepotential.

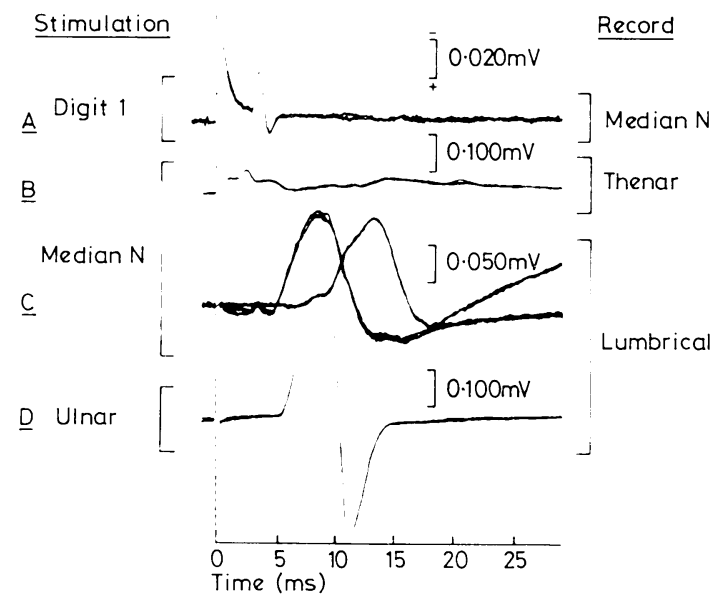

Fig 6 Illustration taken from a patient with motor neuron disease $(A)$. A normal orthodromic median nerve sensory nerve action potential could be recorded at the level of the wrist in response to stimulation of the first digit $(B)$. Supramaximal median nerve stimulation at the level of the wrist evoked a surface positive response over the thenar muscles beginning at approximately $4 \mathrm{~ms}$ and preceded by a sensory prepotential $(C)$. The corresponding "sink" for the thenar surface positive response was $L_{1}$ and $L_{2}$ (surface negative). Here the median nerve has been stimulated at the wrist and elbow. Here too, the " $M$ " response to wrist stimulation was preceded by a low voltage sensory prepotential. At the same $L_{1}$ and $L_{2}$ surface location a much larger potential was recorded in response to supramaximal ulnar nerve stimulation.

\section{Discussion}

This investigation has established that at a late stage in the entrapment of the median nerve beneath the flexor retinaculum, the median nerve contribution to the innervation to the lumbrical muscles may be relatively preserved in relation to the median nerve innervation of the thenar muscles. In those patients in whom only a positive response was detected on the surface of the thenar muscles, the electrogenic origin was invariably the lumbrical muscles innervated by the median nerve. Even when the earlier surface positive was followed by a negative response, the earlier positive corresponded to the shorter latency lumbrical electrogenic source. This curious relative preservation of lumbrical motor fibres may be a consequence of a more protected position of the lumbrical motor fibres in the median nerve at the level of the flexor retinaculum. This suggested protected position may account for the protection from the mechanical or even ischaemic factors responsible for the median nerve injury in this entrapment. ${ }^{8}$

There is anatomical and physiological support for the postulated separation of lumbrical and thenar motor fibres in the median nerve. First, the lumbrical muscles may be spared relative to the thenar muscles when the median nerve is partially transected at or just proximal to the wrist. This suggests that the lumbrical fascicle occupies a more dorsal position in the median nerve at the wrist. $^{2}$ Second, electrical stimulation of the median nerve, directly exposed at operation, has shown that the lowest threshold region for excitation of the lumbrical muscles is over the dorsal surface of the median nerve whereas the ventral surface is the lowest threshold region for the thenar group. The sparing of the lumbrical innervation is relative only. At the late stage of the median neuropathies reported here, there were frequently only a few lumbrical motor units left, although there were examples where the lumbrical innervation was much better preserved.

In the more proximal lesions, for example the C5-6 disc protrusion and thoracic inlet compressions, either no lumbrical sparing was present or only a few lumbrical motor fibres survived when there was total denervation of the thenar muscles innervated by the median nerve. Therefore, at the level of the brachial plexus, ventral roots or spinal cord, the motor fibres to the lumbrical and thenar muscle groups are probably equally vulnerable to injury. The mechanism of thenar wasting in a $\mathrm{C} 5-6$ disc protrusion requires some comment because this is a rare consequence of degenerative disc disease at this level. It is possible that with a disc protrusion of this large size, there was ischaemic injury to the ventral spinal cord at the C7 or C8 spinal segment levels because of compression of the radicular arterial contribution to the cord coming in at the C5-6 level. ${ }^{9}$ Unexplained, however, was the cause of the abnormally low voltage sensory nerve action potential recorded from the fifth digit. The last, presumably would require damage to the $\mathrm{C} 8$ posterior root ganglion or the peripheral sensory processes of $\mathrm{C} 8$.

In motor neuron disease the relative preservation of a few lumbrical motor units in the presence of 
total thenar denervation is probably by chance; other patients have total lumbrical denervation and survival of a few median thenar motor units. Equally, chance probably determines in median nerve regeneration following transection and surgical reunion whether the lumbrical or thenar muscles will be the first muscles to be re-innervated. Moreover, lumbrical motor fibres, because of their more dorsal position, may not be transected in partial median nerve transections which totally denervate the thenar muscles.

\section{References}

1 Sunderland S. Nerves and Nerve Injuries. 2nd ed. Edinburgh: Churchill Livingstone, 1978.

2 Perotto AO, Delagi ED. Funicular localisation in partial median nerve injury and the wrists. Arch Phys Med Rehabil 1979;60:165-9.
${ }^{3}$ Brown WF, Hurst LN. Methods to test the outcome of surgical repair of transected human nerves. Symposia, Sixth International Congress of Electromyography in Stockholm, Sweden. Persson A, ed. 1979;121-6.

${ }^{4}$ Gilliatt RW, Le Quesne PM, Logue V, Sumner AJ. Wasting of the hand associated with a cervical rib or band. J Neurol, Neurosurg, Psychiatry 1970;33:615-24.

${ }^{5}$ Gilliatt RW, Willison RG, Dietz V, Williams IR. Peripheral nerve conduction in patients with a cervical rib and band. Ann Neurol 1978;4:124-9.

${ }^{6}$ Buchthal F, Rosenfalck A, Trojaborg W. Electrophysiological findings in entrapment of the median nerve at wrist and elbow. J Neurol, Neurosurg, Psychiatry 1974;37:340-60.

7 Feinstein B, Lindegaard B, Nyman E, Wohlfart G. Morphologic studies of motor units in normal human muscles. Acta Anat (Basel) 1955;23:127-42.

${ }^{8}$ Sunderland $\mathbf{S}$. Nerve lesion in the carpal tunnel syndrome. J Neurol, Neurosurg, Psychiatry 1976;39:615-26.

${ }^{2}$ Hughes JT. In: Blackwood W, Corsellis JAN eds, Greenfields Neuropathology, 3rd ed. London: Edward Arnold, 1976. 